\title{
Head Motion Compensation System for an Office-Based Ear Surgical Device Using Force Control with Disturbance Observer
}

\author{
Wenyu Liang, Kok Kiong Tan \\ Department of Electrical and Computer Engineering, National University of Singapore \\ 4 Engineering Drive 3, 117583, Singapore \\ liangwenyu@nus.edu.sg; kktan@nus.edu.sg
}

\begin{abstract}
When a surgery is shifted from the operating room to the clinic or surgeon's office using the office-based surgical devices, the patient is no longer under general anaesthesia (GA) and the patient's motion can affect the performance of the device and even the success of the surgery. Specifically for an office-based ear surgical device designed to insert a tube on the tympanic membrane, the patient's head motion will affect the success rate of tube insertion. To address this issue, a head motion compensation system based on force control with disturbance observer is proposed and developed in this paper and tested on a mock-up system. The results show that the system estimates the head motion correctly and facilitates the compensation of the motion to maintain a steady contact force.
\end{abstract}

Keywords: motion compensation, surgical device, force control, disturbance observer

\section{Introduction}

Since mechatronic and robotic systems have the capabilities of providing automatic process and accurate fast motions, more and more surgical devices and robots have been designed for assisting the surgeons to carry out the surgeries in a more efficient way. In recent years, there are a number of mechatronic and robotic systems have been developed for surgical applications such as ear surgery [1], oocyte retrieval [2], eye surgery [3], laparoscopic Surgery [4] beating heart surgery [5], minimally invasive surgery [6,7], etc.

Significantly, there is a kind of surgical device/robot named "office-based surgical device" designed for providing/assisting the surgical treatment in clinic or surgeon's office. In [1], an office-based surgical device has been developed, which is designed for carrying out the surgical treatment to the patient with Otitis Media with Effusion (OME) safely and automatically in a single procedure and a short time. OME is a common ear disease arising once there is an accumulation of fluid occurs in the middle ear, which will cause hearing loss, body imbalance, discomfort and reduces the patient's quality of life [8]. The surgical treatment for OME is called myringotomy with tube insertion, which makes an incision on the tympanic membrane (TM) and inserts a tube in the incision so that the fluid can be drained out.

Benefiting from the advantages of the mechatronic system, the ear surgical device can allow office-based myringotomy with tube insertion and thus it has the advantages of avoiding general anaesthesia (GA), costly expertise and equipment, treatment delays. However, when the surgery is shifted from the operating room to the clinic or office by using this device, the patient is no longer under GA. As a result, the patient's head motion will affect the system performance (tube insertion success rate) although the procedure time with the device is very short.

To address the head motion issue, an approach for stabilization is proposed in a previous work [9], combining mechanical restraints and physiological engagement. This approach helps to stabilize the device directly and reduce the patient's head motion, but the mechanical restriction on patient's head may cause the patient discomfort or hurt the patient which is not the best solution.

By revisiting the working process, the tool set of the ear surgical device will bring the tube to touch the patient's tympanic membrane (TM) in the first step. After the touch, if the contact force can be controlled at a certain value then the relative motion between the device and the patient's head or TM can be kept low. Several research works on developing the force control-based motion compensation system for medical and surgical devices have been reported in [5] and [10][12], which shows the contact force control can facilitate the motion compensation. However, the response of the force 
control system may not be fast enough in some applications [5]. Hence, another approach for stabilization is proposed in [13] using force and vision feedback which shows a good performance but a camera system is needed.

The main objective of this paper is to develop a system that can stabilize or compensate the head motion without any additional equipments. To this end, a head motion compensation system using force control with disturbance observer is developed and tested in this paper. The rest of this paper is organized as follows. First, the ear surgical device is introduced in section 2. Next, the details of the head motion compensation system are presented in section 3 . In section 4, the system is tested and the experimental results on a mock-up system are discussed. Finally, the conclusions are drawn in section 5.

\section{System Description}

The setup of the ear surgical device is shown in Fig. 1. It mainly consists of two parts: main device and support arm.

In the main device, a single-axis linear ultrasonic motor (USM) stage for controlling the tool set movements (cutter and holder) along Z-axis and a cutter retraction (CR) mechanism for controlling the cutter movements are employed. A highly sensitive force sensor is embedded for measuring the contact force.

For the support arm, it is a 7-DOF universal arm with a lock for assisting the surgeon to stabilize the device.

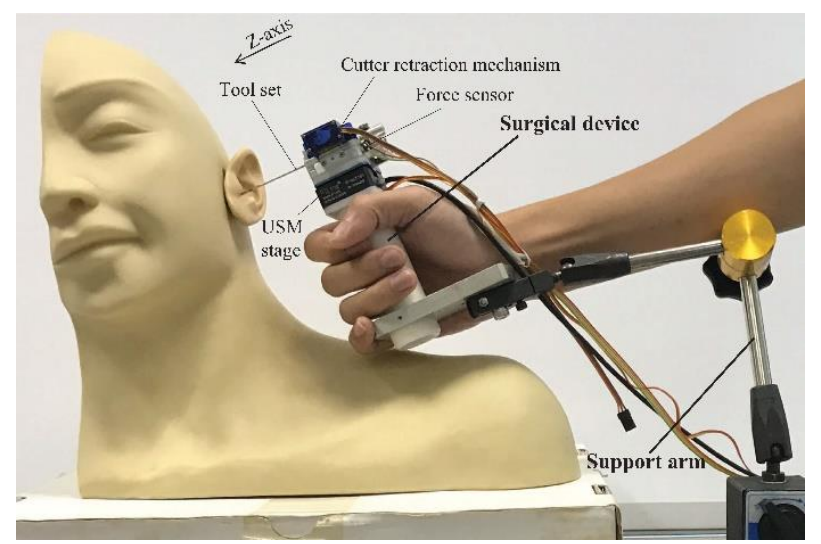

Fig. 1: Setup of the ear surgical device for OME.

The working process of the surgical device is shown in Fig. 2, which generally has five steps:

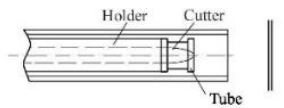

(a) Initialization

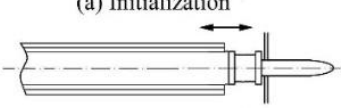

(c) Myringotomy

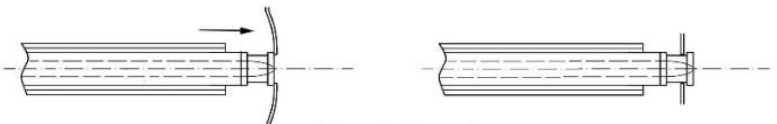

(d) Tube insertion

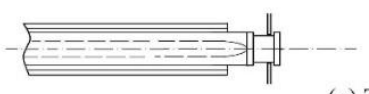

(e) Tube release

(b) Touch detection

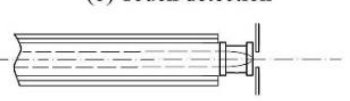

$$
\text { (n) }
$$



TM;

(c) Myringotomy: pushing out the cutter from the holder (driven by the CR mechanism) to make an incision on the

(d) Tube insertion: retracting the cutter just inside the tube (driven by the CR mechanism) and inserting the tube by the holder (driven by the USM stage);

(e) Tube release: retracting the cutter fully inside the holder and withdrawing the tool set (driven by the USM stage).

Step (b) to (e) are accomplished efficiently and automatically by the surgical device. After step (b), the relative motion and the contact force between the tube (on the tool set) and the TM are required to be within a small threshold so that a high success rate can be ensured [13]. Due to the usage of the support arm and the linkage between the TM and head, the relative motion and the contact force is only affected by the head motion. It is thus important that the head motion can be compensated during the working process.

\section{Head Motion Compensation}

As mentioned, contact force control can help to stabilize the relative motion between the device and the object. Moreover, the head motion $(d)$ can be considered as an external disturbance to the device. Lumping it together with the uncertainty $\left(\Delta P_{f}\right)$ due to the slight differences among different TMs, they are treated as a lumped disturbance $\left(d_{l}\right)$, which can be estimated by a disturbance observer (DOB) [14]. To compensate this disturbance, a control scheme shown in Fig. 3 is proposed. It comprises the force/position controller proposed in [15] plus the DOB-based motion compensation.

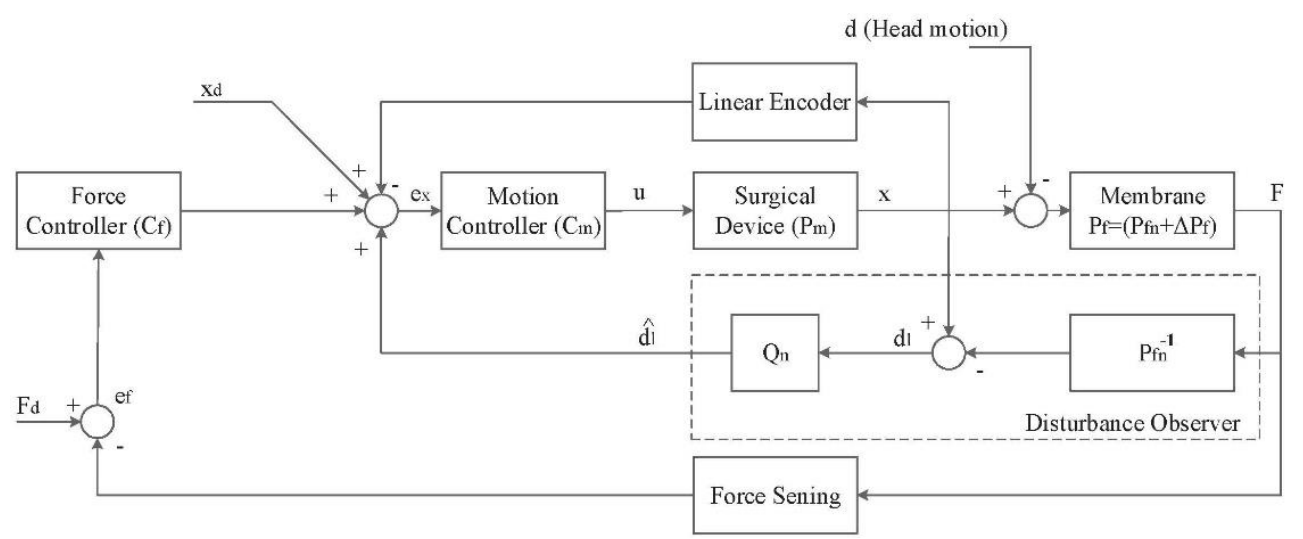

Fig. 3: Control scheme of the head motion compensation system.

\subsection{Force/Position Controller}

Since the relative position $(x)$ between the tube and the TM is related to the contact force $(F)$, the contact force control can be achieved by controlling the relative position. The force/position controller uses a cascade control structure, which has a motion controller in the inner loop for controlling the USM stage's position precisely and a force controller in the outer loop for maintaining the contact force at a desired value.

The motion controller referring to [15] is a composite controller given by (1). A PID (Proportional-IntegralDerivative) controller tuned by the LQR (Linear-Quadratic Regulator) technique is used as the main controller. A modelbased adaptive friction compensation, including an asymmetric Coulomb friction model-based compensation and a sliding mode controller, is designed to compensate the nonlinearity and eliminate the uncertainty.

$$
\begin{gathered}
u_{m}(t)=K_{p m} e_{x}(t)+K_{i m} \int_{0}^{t} e_{x}(\tau) d \tau+K_{d m} \frac{d e_{x}(t)}{d t} \\
+[\sigma \operatorname{sign}(\dot{x})-\delta|\operatorname{sign}(\dot{x})|]+\hat{k}_{s} \operatorname{sign}\left(E_{x}(t)^{T} P B\right)
\end{gathered}
$$

where $e_{x}$ is the position error, $E_{x}$ contains the error states, $B, \sigma, \delta$ are the system parameters, $P$ is the solution of the Riccati equation while calculating the PID parameters: $K_{p m}=40.5069, K_{i m}=160, K_{d m}=0.0994$, $\hat{k}_{s}=\operatorname{Proj}\left(\rho_{1}\left|E_{\chi}^{T} P B\right|, \hat{k}_{s}\right), \rho_{1}$ is the adaptive gain and $\operatorname{Proj}(\cdot)$ is the smooth projection algorithm. 
Considering that the friction compensation will cancel most nonlinear part of the USM stage, the PID controller mainly deals with the linear part. Thus, the position closed-loop system can be simplified to (2).

$$
G_{m}(s)=\frac{C_{m}(s) P_{m}(s)}{1+C_{m}(s) P_{m}(s)}
$$

where $P_{m}(s)$ is the linear model of the USM stage and $C_{m}(s)$ is the PID controller:

$$
C_{m}(s)=\frac{U_{m}(s)}{E_{x}(s)}=K_{p m}+K_{i m} \frac{1}{s}+K_{d m} s
$$

The force controller is also a PID controller, but to reduce the undesirable noise amplification brought by the pure derivation action, the pure derivative action is combined with a first-order low-pass filter to form the PIDF (PID with filter) controller:

$$
C_{f}(s)=\frac{U_{f}(s)}{E_{f}(s)}=K_{p f}+K_{i f} \frac{1}{s}+K_{d f} \frac{s}{T_{f} s+1}
$$

Thus, the outer closed-loop system can be represented by (5).

$$
G_{f}(s)=\frac{C_{f}(s) G_{m}(s) P_{f}(s)}{1+C_{f}(s) G_{m}(s) P_{f}(s)}
$$

where $E_{f}(s)$ is the force error, $P_{f}(s)=\frac{F(s)}{X(s)}$ represents the relation between the contact and the relative position between the tube and the TM, $K_{p f}=0.58, K_{i f}=6.72, K_{d f}=0.00724, T_{f}=0.0171$ are the PIDF controller parameters.

\subsection{Disturbance Observer-Based Motion Compensation}

For the lumped disturbance, it can be indicated by (6).

$$
D_{l}(s)=D(s)+\left[P_{f}^{-1}(s)-P_{f n}^{-1}(s)\right] F(s)
$$

where the disturbance induced from head motion $D(s)$ is the dominant part of $D_{l}(s)$ in this application (i.e., $D_{l}(s) \approx D(s)$ while $\left.P_{f}(s) \approx P_{f n}(s)\right), P_{f n}(s)$ is the nominal model without the disturbance and $P_{f n}^{-1}(s)$ is the inverse of the nominal model.

For the disturbance observer, referring to Fig. 3, since $F(s)=P_{f}(s)[X(s)-D(s)]$, it is easy to show that the disturbance can be estimated by

$$
D(s)=X(s)-P_{f}^{-1}(s) F(s)
$$

Substituting (7) to (6), we can have

$$
\begin{gathered}
D_{l}(s)=X(s)-P_{f}^{-1}(s) F(s)+\left[P_{f}^{-1}(s)-P_{f n}^{-1}(s)\right] F(s) \\
=X(s)-P_{f n}^{-1}(s) F(s)
\end{gathered}
$$


As can be seen, $X(s)$ and $F(s)$ are measurable and $P_{f n}(s)$ is defined and identified in [15] which shows $P_{f n}(s)=$ $\frac{24.27 s+35.87}{s^{2}+7.683 s+19}$, so the lumped disturbance can be obtained and calculated from (8).

However, the relative degree of $P_{f n}^{-1}(s)$ is less than zero, i.e., $P_{f n}^{-1}(s)$ is not proper. To make the DOB proper, a Qfilter (represented by $Q(s)$ ) which normally is a low-pass filter with its relative degree higher than that of $P_{f n}^{-1}(s)$ is applied. Then, the estimation of $D_{l}(s)$ is given by (9), which will be fed back to the outer loop of the force/position controller for the head motion compensation since controlling the USM stage is the only way to change the relative position between tool set and TM.

$$
\widehat{D}_{l}(s)=Q(s) X(s)-Q(s) P_{f n}^{-1}(s) F(s)
$$

For this application, $Q(s)$ uses a second-order low-pass filter as shown below.

$$
Q(s)=\frac{1}{\frac{s^{2}}{\omega_{n}^{2}}+\frac{s}{q \omega_{n}}+1}
$$

where $\omega_{n}=2 \pi f_{n}, f_{n}$ is the natural frequency, $q$ is the quality factor. Define $f_{c}$ as the cutoff frequency, it has $f_{c}=f_{n}$ when $q=1 / \sqrt{2}$, i.e., it becomes second-order Butterworth low-pass filter.

At the frequency less than the cutoff frequency of the low-pass filter, $Q(s) \approx 1$ and then $\widehat{D}_{l}(s) \approx D_{l}(s)$; otherwise, $Q(s) \approx 0$ and the filter cuts off the signal (i.e., $\widehat{D}_{l}(s)=0$ ). It is important that the Q-filter is designed properly so that the disturbance estimation is fast enough for the application. To design the Q-filter in this application, the two remarks presented below provide some guidelines.

Remark 1: The cutoff frequency $\left(f_{c}\right)$ of the Q-filter must be larger than the bandwidth $\left(\Delta f_{d}\right)$ of the external disturbance (i.e., $f_{c}>\Delta f_{d}$ ).

Remark 2: The cutoff frequency $\left(f_{c}\right)$ of the Q-filter should not be greater than the gain crossover frequency $\left(f_{\text {gmc }}\right)$ of the position closed-loop control system $G_{m}(s)$ (i.e., $f_{c}<f_{g m c}$ ).

For Remark 1, it is to ensure that the head motion can be estimated correctly in its frequency range.

For Remark 2, since the proposed DOB-based motion compensation is different from the conventional DOB-based compensation method in which the estimated disturbance is fed back to the inner loop (to the system input) directly, the proposed compensation method requires a condition that the output of $G_{m}(s)$ should be as close as possible to the input of $G_{m}(s)$ (i.e., $G_{m}(s) \approx 1$ ). To meet this condition, the input to $G_{m}(s)$ should be within the bandwidth which is below the gain crossover frequency of $G_{m}(s)$ (because $G_{m}(j \omega) \approx 1, \forall \omega<2 \pi f_{g m c}$ ).

\section{Experiments and Results}

To validate the performance of the proposed head motion compensation system, an experimental setup is used as shown in Fig. 4. A mock-up system, consisting of a Poly Ethylene (PE) mock membrane with similar characteristics to the TM and another USM stage for providing the simulated head motion, is set up and used in the tests.

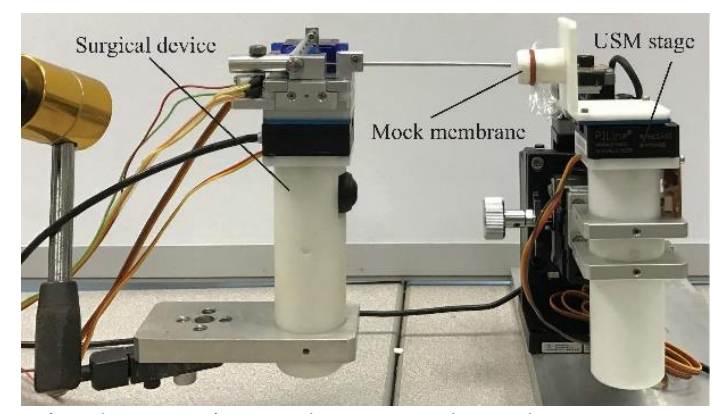

Fig. 4: Experimental setup and mock-up system. 
From [13], it is reported that the human head motion is within the bandwidth of about $0.1 \mathrm{~Hz}$ and the maximum amplitude of $\pm 1 \mathrm{~mm}$ when the patient is in a proper lying position. Based on Remark 1 and Remark 2, the Q-filter parameters are chosen as $f_{n}=4 \mathrm{~Hz}$ and $q=0.707$. The desired contact force is $0.04 \mathrm{~N}$.

During the tests, different sine wave motions are used as the simulated head motions. Table 1 shows the maximum absolute error and the root-mean-square (RMS) error of the disturbance estimation (where the estimated error is defined as $\bar{d}=\hat{d}_{l}-d_{m}, d_{m}$ is the simulated head motion which can be given by the linear encoder on second USM stage). As can be seen, the estimated disturbance can match the actual disturbance correctly. The accuracy is more than $90 \%$ and the small difference may be due to the system uncertainty (which is not included in the estimated error).

Table 1: Disturbance estimated error by the DOB.

\begin{tabular}{|c|l|c|c|c|c|c|c|}
\hline \multirow{2}{*}{ Sine wave } & Frequency & $0.05 \mathrm{~Hz}$ & $0.05 \mathrm{~Hz}$ & $0.1 \mathrm{~Hz}$ & $0.1 \mathrm{~Hz}$ & $0.2 \mathrm{~Hz}$ & $0.2 \mathrm{~Hz}$ \\
\cline { 2 - 7 } & Amplitude & $0.5 \mathrm{~mm}$ & $1.0 \mathrm{~mm}$ & $0.5 \mathrm{~mm}$ & $1.0 \mathrm{~mm}$ & $0.5 \mathrm{~mm}$ & $1.0 \mathrm{~mm}$ \\
\hline $\max (|\bar{d}|)$ & $24.6 \mu \mathrm{m}$ & $32.6 \mu \mathrm{m}$ & $32.5 \mu \mathrm{m}$ & $66.8 \mu \mathrm{m}$ & $47.3 \mu \mathrm{m}$ & $83.2 \mu \mathrm{m}$ \\
\hline \multicolumn{2}{|c|}{$\operatorname{RMS}(\bar{d})$} & $13.2 \mu \mathrm{m}$ & $18.8 \mu \mathrm{m}$ & $18.7 \mu \mathrm{m}$ & $33.0 \mu \mathrm{m}$ & $29.5 \mu \mathrm{m}$ & $54.1 \mu \mathrm{m}$ \\
\hline
\end{tabular}

Table 2: Comparisons between the force control with and without DOB.

\begin{tabular}{|c|c|c|c|c|c|}
\hline \multicolumn{2}{|c|}{ Sine wave } & \multicolumn{2}{c|}{ Force control only } & \multicolumn{2}{c|}{ Force control with DOB } \\
\hline Frequency & Amplitude & $\max (|\bar{d}|)$ & $\operatorname{RMS}(\bar{d})$ & $\max (|\bar{d}|)$ & $\operatorname{RMS}(\bar{d})$ \\
\hline $0.05 \mathrm{~Hz}$ & $0.5 \mathrm{~mm}$ & $0.0087 \mathrm{~N}$ & $0.0051 \mathrm{~N}$ & $0.0054 \mathrm{~N}$ & $0.0016 \mathrm{~N}$ \\
\hline $0.05 \mathrm{~Hz}$ & $1.0 \mathrm{~mm}$ & $0.0121 \mathrm{~N}$ & $0.0083 \mathrm{~N}$ & $0.0102 \mathrm{~N}$ & $0.0020 \mathrm{~N}$ \\
\hline $0.1 \mathrm{~Hz}$ & $0.5 \mathrm{~mm}$ & $0.0123 \mathrm{~N}$ & $0.0081 \mathrm{~N}$ & $0.0106 \mathrm{~N}$ & $0.0023 \mathrm{~N}$ \\
\hline $0.1 \mathrm{~Hz}$ & $1.0 \mathrm{~mm}$ & $0.0212 \mathrm{~N}$ & $0.0146 \mathrm{~N}$ & $0.0140 \mathrm{~N}$ & $0.0027 \mathrm{~N}$ \\
\hline $0.2 \mathrm{~Hz}$ & $0.5 \mathrm{~mm}$ & $0.0201 \mathrm{~N}$ & $0.0141 \mathrm{~N}$ & $0.0129 \mathrm{~N}$ & $0.0033 \mathrm{~N}$ \\
\hline $0.2 \mathrm{~Hz}$ & $1.0 \mathrm{~mm}$ & $0.0386 \mathrm{~N}$ & $0.0267 \mathrm{~N}$ & $0.0194 \mathrm{~N}$ & $0.0044 \mathrm{~N}$ \\
\hline
\end{tabular}

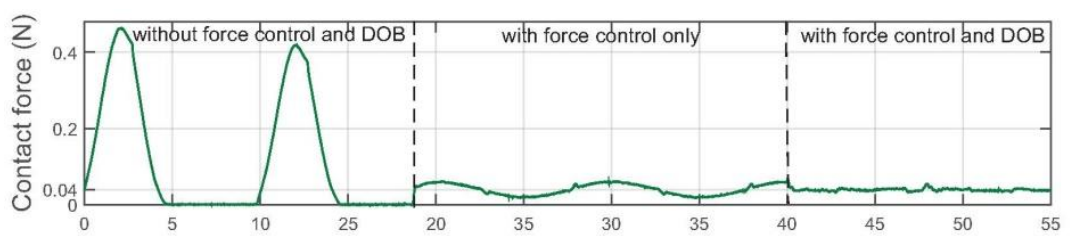

(a) Comparison among different methods

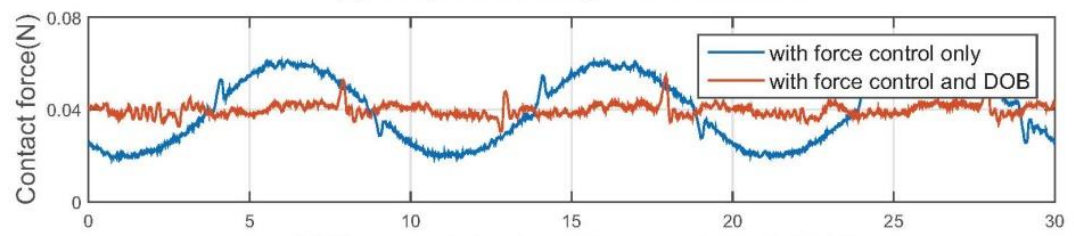

(b) Force control only vs. Force control with DOB

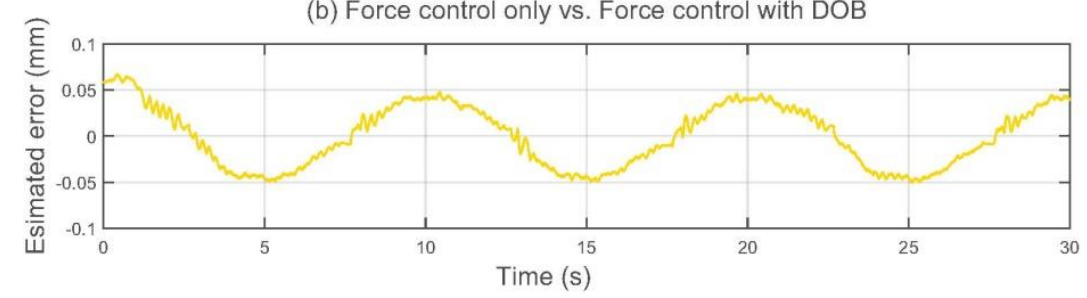

(c) Estimated disturbance error

Fig. 5: System performance at $0.1 \mathrm{~Hz}, \pm 1 \mathrm{~mm}$ sine wave motion. 
Table 2 shows the force error information of the force control with and without DOB under different motions. Fig. 5 shows the system performance with different methods when the sine wave motion frequency is $0.1 \mathrm{~Hz}$ and amplitude is $\pm 1 \mathrm{~mm}$. As can be seen, the contact force cannot be kept at the desired value without the force control. Once the force control is applied, the force can be controlled but it is still affected by the disturbance. After the DOB-based motion compensation is added, the force errors are greatly reduced (RMS errors are reduced by 68\% to 83\%) for all disturbed motions.

\section{Conclusion}

In this paper, a head motion compensation system based on force control plus DOB-based motion compensation is proposed, developed and tested. To validate the performance of the proposed system, a mock-up system simulating the TM and the head motion is used. The experimental results show that the DOB can estimate the head motion correctly and the proposed system can greatly compensate the head motion. Thus, the contact force and the relative position between the tool set and the TM can be maintained within a small range.

Furthermore, some future works are shown as follows. The real human head motion data can be input to the mock-up system for the performance validation. The adaptive and learning control algorithms can be also considered in this system to improve the motion compensation performance.

\section{Acknowledgements}

This research was supported by the Biomedical Engineering Programme (BEP) of the Science and Engineering Research Council (SERC), Singapore (SERC Grant No.: 132148 0014).

\section{References}

[1] K. K. Tan, W. Liang, P. L. Phuong, S. Huang, C. W. Gan, and H. Y. Lim, "Design of a surgical device for officebased myringotomy and grommet insertion for patients with otitis media with effusion," ASME Journal of Medical Devices, vol. 8, pp. 031001-031001-12, 2014.

[2] K. K. Tan, S. Huang, M. H. T. Nguyen, W. Liang, and S. C. Ng, "An innovative design for in-vitro fertilization oocyte retrieval systems," IEEE Transactions on Industrial Informatics, vol. 9, no. 4, pp. 1892-1899, 2013.

[3] T. Ueta, Y. Yamaguchi, Y. Shirakawa, T. Nakano, R. Ideta, Y. Noda, A. Morita, R. Mochizuki, N. Sugita, M. Mitsuishi, and Y. Tamaki, "Robot-assisted vitreoretinal surgery: Development of a prototype and feasibility studies in an animal model," Ophthalmology, vol. 116, no. 8, pp. 1538-1543.e2, 2009.

[4] H. Choi, H. S. Kwak, Y. A. Lim, and H. J. Kim, "Surgical robot for single-incision laparoscopic surgery," IEEE Transactions on Biomedical Engineering, vol. 61, no. 9, pp. 2458-2466, 2014.

[5] S. G. Yuen, D. P. Perrin, N. V. Vasilyev, P. J. d. Nido, and R. D. Howe, "Force tracking with feed-forward motion estimation for beating heart surgery," IEEE Transactions on Robotics, vol. 26, no. 5, pp. 888-896, 2010.

[6] "Introduction to robot-assisted minimally invasive surgery (MIS)," P. Gomes, editor, Medical Robotics, Woodhead Publishing, 2012.

[7] D. Pisla, B. Gherman, C. Vaida, M. Suciu, and N. Plitea, "An active hybrid parallel robot for minimally invasive surgery," Robotics and Computer-Integrated Manufacturing, vol. 29, no. 4, pp. 203-221, 2013.

[8] R. D. Eredità, R. R. Marsh, S. Lora, and K. Kazahaya, "A new absorbable pressure-equalizing tube," Otolaryngology-Head and Neck Surgery, vol. 127, no. 1, pp. 67-72, 2002.

[9] K. K. Tan, W. Liang, T. H. Lee, C. H. Choy, and Z. Shen, "Design and development of a feedback mechanism and approach for patient-instrument stabilization during office-based medical procedures," in ICST2013, pp. 520-525, 2013.

[10] W. T. Latt, R. C. Newton, M. Visentini-Scarzanella, C. J. Payne, D. P. Noonan, J. Shang, and G. Z. Yang, "A handheld instrument to maintain steady tissue contact during probe-based confocal laser endomicroscopy," IEEE Transactions on Biomedical Engineering, vol. 58, no. 9, pp. 2694-2703, 2011.

[11] S. Kyeong, D. Chang, Y. Kim, G. M. Gu, S. Lee, S. Jeong, and J. Kim, "A hand-held micro surgical device for contact force regulation against involuntary movements," In EMBC2015, pp. 869-872, 2015.

[12] J. M. Florez, J. Szewczyk, and G. Morel, "An impedance control strategy for a hand-held instrument to compensate for physiological motion,” in ICRA2012, pp. 1952-1957, 2012. 
[13] W. Liang, W. Gao, and K. K. Tan, "Stabilization system on an office-based ear surgical device by force and vision feedback," Mechatronics, vol. 42, pp. 1-10, 2017.

[14] W. H. Chen, J. Yang, L. Guo, and S. Li, "Disturbance-observer-based control and related methods: An overview," IEEE Transaction on Industrial Electronics, vol. 63, no. 2, pp. 1083-1095, 2016.

[15] W. Liang and K. K. Tan, "Force feedback control assisted tympanostomy tube insertion," IEEE Transaction on Control Systems Technology, vol. 45, no. 3, pp. 1007-1018, 2017. 